\title{
Medical Affairs Transformation in Specialty Pharma: Next-Level Collaboration at the Core
}

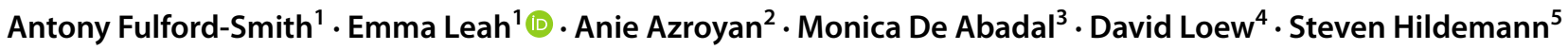

Accepted: 23 January 2022 / Published online: 6 March 2022

(c) The Author(s) 2022

\begin{abstract}
Pharmaceutical companies with a medical mindset and an empowered Medical Affairs function are well equipped to meet the needs and expectations of patients and society. Yet, as capacity to understand and serve those needs accelerates, so too do expectations. Evidence-based practice, without delay, is expected throughout the development and delivery of medicine, healthcare, and information, and potential sources of evidence are legion. To keep pace and go beyond, to innovate, requires efficiencies. Not the disguised cutbacks of political language, but the collaborative constructive efficiencies of shared learning, forming new evidence bases for further progress. Here, we describe the first year of a medical transformation process at a global mid-sized pharmaceutical company. Beginning with a broad review designed to leverage collective intelligence and focus on meaningful outcomes for patients, this process examined and reshaped the structure, culture, and tools of the medical organization and its interactions within and outside the company. We report the findings of the diagnostic phase, outline the solutions implemented to date, and anticipate the next steps in this dynamic evolving journey.
\end{abstract}

\section{Key Points}

The multi-faceted strategic role of Medical Affairs is increasingly recognized and relied on within the pharmaceutical industry and healthcare systems.

To effectively harness medical expertise in the data age and make measurable improvements to patients' lives requires new metrics and adaptive nimble approaches.

Optimizing the flow of knowledge to and from Medical Affairs professionals empowers them to co-create solutions and achieve meaningful outcomes for patients.
Antony Fulford-Smith

antonyfs18@gmail.com

1 Chief Medical Office, Ipsen, UK

2 Chief Medical Office, Ipsen, France

3 North America Medical Affairs, Ipsen, USA

4 Chief Executive Officer, Ipsen, France

5 Chief Medical Officer, Ipsen, France

\section{Introduction}

An increasingly clear vision of near-future specialty pharma (developing medicines predominantly prescribed or initiated by hospital specialists), in which Medical Affairs harnesses the power of the data age, is described in industry and professional society articles [1-8], and peer-reviewed articles [9-13]. With confident yet forward looking leadership and collaborative practice, a digitally empowered medical organization will direct and translate internal and external resources to serve the needs and expectations of patients and healthcare professionals (HCPs), within the pragmatic realities of healthcare systems. Achieving meaningfully improved health outcomes for patients will be at the core. Having evolved from a support function within historically more consumer-based organizations, through a bridge, to a strategic pillar, from reactive to proactive, transforming in response to external pressures and propelled by unprecedented global change and therapeutic advances, medical functions will nimbly deploy novel yet validated approaches to co-create better healthcare that is centered on the patient and rooted in evidence. In so doing, they will ensure their deep medical expertise can be impactful in a noisy world. Pharmaceutical companies must remain relevant amidst healthcare-facing innovations from digital giants. So, how? Not in isolation. A clear consensus also highlights the 
importance of developing deep medical capabilities, especially people, generating and leveraging novel insights, and communicating effectively with all relevant stakeholders. The very pace of evolution in Medical Affairs has contributed to challenges in articulating its value, threatening to hamper cross-functional progression [14]. The impact and value of strong medical organizations anywhere should be felt everywhere, to the ultimate benefit of patients and society, if we collaborate at a next level. To that end, we describe a process of medical transformation at a global mid-sized biopharmaceutical company, outlining the initial impetus and steps taken to date, and examining progress toward that near-future vision.

\section{Approach}

\subsection{Collective Transformation}

Ipsen has a 90-year heritage as a family-owned company and publicly traded business, with an accompanying legacy; of experience, reputation, and relationships, and also of historical perspective and systems. Prior to Q2/2020, Medical Affairs supported internal decision making, but a cultural shift was needed to position the medical organization as a strategic driver of innovation and value alongside research and development, commercial, and market-access functions $[1,2]$. This positioning, which reflects the emerging industry-wide vision of medical affairs as we introduced above, was fundamental to achieve our evolving vision-of medical leadership for patients being at the center of everything

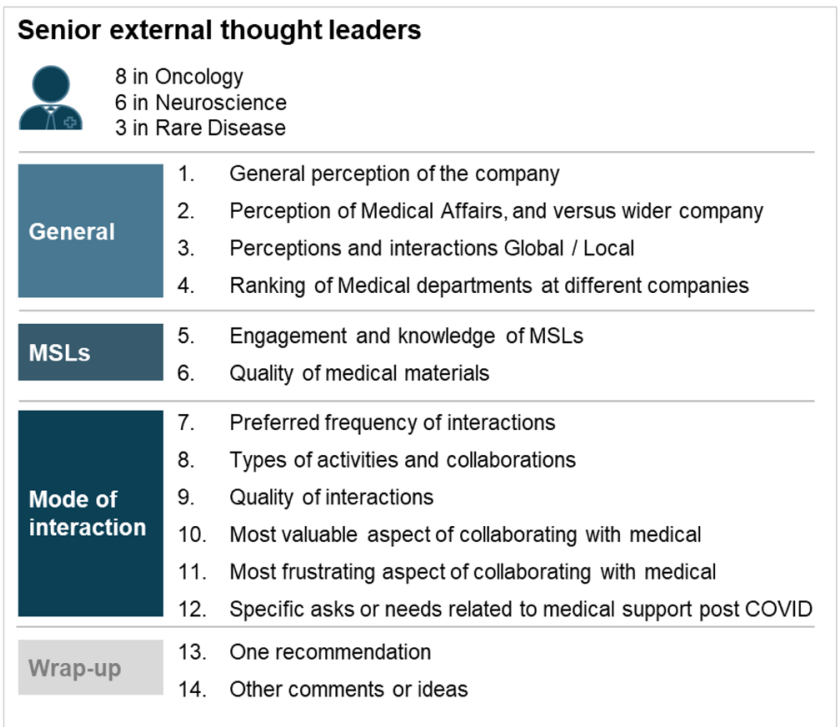

Fig. 1 Scope and dimensions of in-depth interviews during initial benchmarking of medical capabilities. $C M O$ Chief Medical Office, COVID coronavirus disease, ELT Executive Leadership Team, $H C P$ we do. Leading in therapeutic area (TA) strategy allows the voice of data to be more effectively leveraged, not only in translating for and between multiple stakeholders but also in ensuring an evidence basis for resource and development decisions, to deliver value and insights for patients, HCPs, and healthcare systems. In March 2020, a newly appointed Chief Medical Officer joined the Executive Leadership Team, enabling medical to "lead from the C-suite", and a new and empowered Medical Transformation Lead was appointed to further boost medical leadership and ensure deep worldwide implementation. This elevation of medical, essential in reinforcing and executing on its strategic pillar status, was nevertheless a structural tweak. The true cultural shift aimed to leverage collective intelligence throughout the transformative process, and embed this approach within the solutions identified.

\subsection{Diagnosis and Direction}

Rapid initial assessment and benchmarking, facilitated by external consultants, involved representative cross-functional in-depth interviews (Fig. 1); 28 internally and, importantly, 17 with senior external thought leaders with whom Ipsen and key competitors interact, from seven countries and across three TAs. Executive Leadership Team members called for stronger medical partnership in business development, while external interviewees noted that excellent scientific expertise and exchange at a local and global level was hampered by staff turnover and partial under-resourcing. In parallel, an internal survey distributed to 974 staff—global

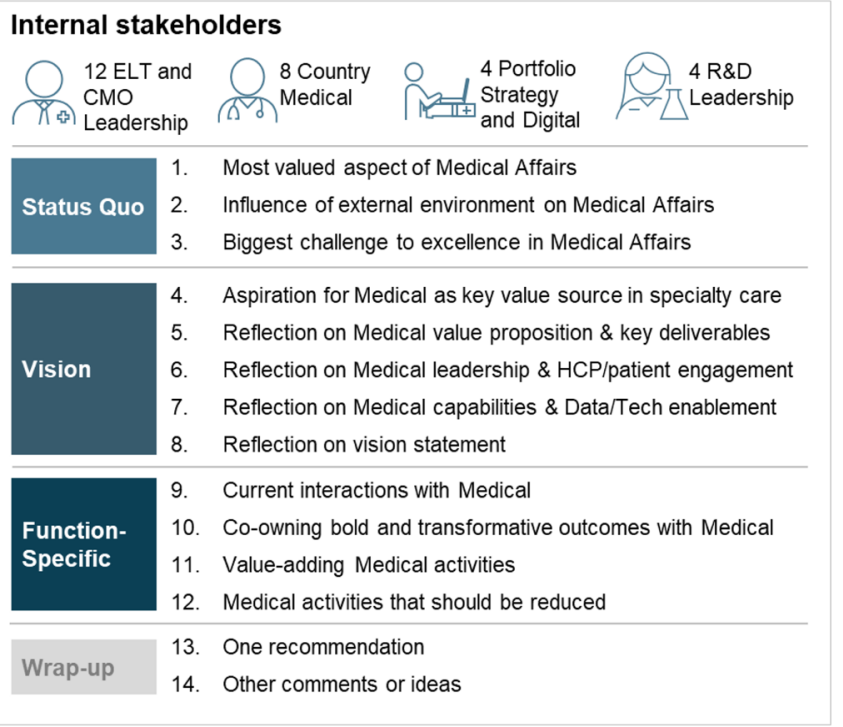

healthcare professional, $M S L$ Medical Science Liaison, $R \& D$ research and development 
leadership team, key partners in other functions, and all medical—generated 725 responses (a 74\% return rate) (Fig. 2). Capabilities in Medical Affairs were perceived as being at or above the industry standard (score of 3.0) in only eight of 18 dimensions assessed. Average perceptions of medical capabilities were higher among medical affairs staff (3.1; $n=378)$ than non-medical affairs staff $(2.7 ; n=347) ; 12$ of the 18 dimensions scored slightly above average among the former. Overall, 'traditional' areas of expertise, including compliance, publications, and medical information and education rated above standard, contrasting with crucial data-centered capabilities including performance tracking, real-world evidence (RWE), and digital and analytics (Fig. 2). The Medical Science Liaison (MSL) and training dimensions scored above or at standard but were acknowledged as being of critical importance for the future impact of the medical organization on patient care. Similarly, it was a priority to strengthen the existing and well-regarded Patient Affairs function. During this phase, the strategic direction of medical transformation was set according to overarching aims and fully embraced by Ipsen's CEO and Executive Leadership Team, to: measurably improve patients' lives along the patient journey, as well as HCP experiences; demonstrate medical leadership; advance medical care in long-term collaborations; be at the leading edge of (data and technology enabled) innovation in specialty care; and attract, develop, and retain leading talent.

\subsection{Prescription}

Five priority areas for change were identified and six workstreams created (Fig. 2), each with a medical lead. These owners formed a medical transformation leadership team, responsible for driving and monitoring overall progress. Individual workstreams included Executive Leadership Team-nominated partner owners from other relevant functions and geographies. Priorities, set with multi-year horizons, included specific nearterm goals within 3-month and 6-month timelines. Changes enacted alongside the rollout of the transformation roadmap included seeking to fill immediate capability gaps: medical professionals with experience in business development, digital professionals with medical experience, and training and knowledge transfer leads. Other re-structuring involved mirroring in the medical organization the structure of TAs as established in the research and development organization, and the international structure of the commercial organization. Within Global Medical Affairs TAs, capabilities were strengthened for external innovation and business development. Medical Excellence, expanded with new training and knowledge transfer roles per TA, was joined within Global Medical Affairs Capabilities by a novel Data, Insights \& Strategy function led by an experienced RWE specialist, incorporating the existing Biometry team. Global Medical Affairs Regional was boosted with new regional heads to drive global and local alignment. Patient safety was fully integrated in the
Aggregated results (average per dimension)

\begin{tabular}{l|c} 
Dimension & 3 \\
\hline Compliance & 3.6 \\
\hline Publications & 3.2 \\
\hline Events and Congresses & 3.2 \\
\hline Medical Info & 3.1 \\
\hline Ad Boards & 3.1 \\
\hline Medical Education & 3.1 \\
\hline MSL & 3.1 \\
\hline Value-based & 3.1 \\
\hline Stakeholder & 3.0 \\
\hline Medical Strategy & 3.0 \\
\hline Talent & 3.0 \\
\hline HEOR & 2.9 \\
\hline ISS & 2.9 \\
\hline Performance & 2.8 \\
\hline Data/evidence generation & 2.8 \\
\hline Phase 3b/4 trials & 2.8 \\
\hline RWE & 2.6 \\
\hline Digital and Analytics & 2.4 \\
\hline
\end{tabular}

\section{Survey demographics}

\begin{tabular}{|c|c|}
\hline & 974 people contacted \\
\hline 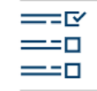 & 725 responses $(74 \%)$ \\
\hline & Global: 226 \\
\hline & Europe: 297 \\
\hline & North America: 91 \\
\hline & Asia-Pacific / China: 68 \\
\hline & Other: 43 \\
\hline & Medical: 378 \\
\hline & R\&D: 65 \\
\hline & Commercial: 145 \\
\hline & Other: 137 \\
\hline
\end{tabular}

Fig. 2 Medical capabilities benchmarking survey. Score of $3=$ industry-standard; 5 = best-in-class. Conducted April-May 2020. Ad advisory, HEOR health-economic outcomes research, ISS investigator- sponsored study, MSL Medical Science Liaison, $R \& D$ research and development, $R W E$ real-world evidence 
Chief Medical Officer organization retaining a separate dedicated global structure. The voice of the patient was boosted per TA with new resources in Patient Affairs, and a new Global MSL Lead role completed the new medical structure, established after 3 months by late 2020 .

A detailed transformation roadmap was rolled out via a dedicated global townhall in September 2020, followed by supportive regional townhalls and cross-functional regional implementation team kick-off meetings to ensure full worldwide implementation. Each workstream included specific goals, activities, and best-practice definitions (Fig. 3), with progress to be monitored via key performance indicators (KPIs). Important in the new culture was that KPIs assessed meaningful metrics [15]; the impact as well as the magnitude of an activity. For example, within the stakeholder engagement workstream, operational KPIs, such as the rollout of a new tool for collecting insights and the percentage of users accessing it, were accompanied by performance KPIs such as the proportions of countries sharing and applying insights, and stakeholder feedback on the usefulness of gathered insights. Into early 2021, localization of the roadmap was driven through executive-sponsored medical health checks with country managers and medical directors to identify capability and resource gaps.

\section{Outcomes}

\subsection{People: Careers, Training, and Knowledge Transfer}

A new medical career path tool allows staff across diverse functions to explore how their expertise can be applied and developed in well-trodden or unique directions. Roles have been mapped within a competency framework, allowing lateral and vertical moves to be plotted, boosting progression opportunities and illustrating the interconnectedness of functions and flow of skills within the organization. New recruitment and fast-track promotion programs have been instigated to encourage attraction and retention of the best talent. Medical-Science Liaison and patient centricity teams have been expanded. Training has been updated to create the Medical Excellence Academy, to systematically train medical staff on core and role-specific capabilities. Integral to this plan has been the hiring of new Knowledge Transfer Leads per TA, to ensure a deep understanding of products, TA, and other critical medical information for all relevant staff. These roles proved challenging to fill, as few corollaries exist, but are rapidly making an impact. As new core training modules come online, the team is working with colleagues in TAs, publications, communications, and medical information to pilot effective ways of providing real-time updates, such as priority data at congresses, new manuscripts, and collective insights. Periodic assessment will measure skill development against defined pillars, and delivery of content in terms of scientific excellence, clarity, and objectivity. This structure will be important to equip staff, and also to ensure staff feel equipped, to provide value in their interactions. Expansion of the medical affairs remit has prompted some industry voices to call for the creation of "super-medics" [16]. Others caution against creating a sense that people must be superhuman to manage emerging technologies and new approaches [17]. Using data-driven approaches to foster both individual development and collaborative expertise will help embed the new organizational culture while protecting and leveraging a bedrock of high-functioning talent.

\begin{tabular}{|c|c|c|}
\hline \multicolumn{2}{|c|}{ Priority areas } & Workstreams \\
\hline & $\begin{array}{l}\text { Talent, Organization \& } \\
\text { Governance }\end{array}$ & $\begin{array}{l}\text { Medical career paths, attraction \& retention, } \\
\text { Organization \& Governance alignment }\end{array}$ \\
\hline & $\begin{array}{l}\text { Training \& knowledge } \\
\text { transfer }\end{array}$ & Scientific content, delivery \& assessment \\
\hline & & Stakeholder engagement \& Medical Insights \\
\hline & & Medical Launch readiness \\
\hline 正 & $\begin{array}{l}\text { Quality upgrade for } \\
\text { current study landscape }\end{array}$ & $\begin{array}{l}\text { Gap Analysis of all current studies \& } \\
\text { programs }\end{array}$ \\
\hline & $\begin{array}{l}\text { Data/analytics \& } \\
\text { evidence }\end{array}$ & $\begin{array}{l}\text { Evidence process, strategy \& data } \\
\text { infrastructure }\end{array}$ \\
\hline
\end{tabular}

Fig. 3 Priority areas, workstreams, and best practices incorporated in medical transformation roadmap. Ad advisory, $H C P$ healthcare professional, incl. including, ISS investigator-sponsored study, LCM
Best practice definition

Drive global and local talent and medical capabilities based on a structured competency and career structure with an expanded range of skills (e. omnichannel engagement, data analytics and innovative RWE)

Medical teams consistently demonstrate a diverse core skill set, including: deep medical knowledge, soft skills, business acumen and strategic thinking to partne with commercial and R\&D on strategy development

Broad view of medical influencers with robust TL identification, targeting and engagement (including through digital channels). Highest level of scientific exchange conducted with key TLs, payers, regulators and patient advocates.

Clear, aligned, transparent process in place to ensure consistent, pro-active insight gathering through MSL/HCP stakeholder engagement, Ad boards and Med Info LCM and phase $3 \mathrm{~b} / 4$ plans developed at an early stage (phase 2 or early 3 ) of product development /according to Medical Launch Readiness Framework

Clear standards, processes and systems for different type of studies that ensure and track performance, delivery on strategy and compliance at all times

Medical insights systematically incorporated into an integrated asset evidence plans to identify data gaps that define late phase clinical programs including $3 \mathrm{~b} / 4$, RWE, ISS areas of interest and programs. Clear global RWE analytics strategy using multiple data sources and innovative analytical tools and an "integrated data platform"

lifecycle management, Med Info medical information, MSL Medical Science Liaison, $R \& D$ research and development, $R W E$ real-world evidence, $T L$ thought leader 


\subsection{Stakeholder Engagement}

The upskilling of medical staff, and provision of tailored training materials, are key to effective engagement, enabling the proactive exchange of knowledge, data, and solutions. Another aspect is identifying who to engage with, when, and how to do so most effectively. A key milestone in the medical transformation was achieved in August 2021, with the rollout of the Thought Leader Identification and Mapping tool, a data-driven application to help find, validate, and select thought leaders based on evidence. The tool increases the ease, visibility, and transparency of profile building, and supports planning for local and global activities with HCPs.

\subsection{Medical Insights}

How to gather, analyze, and effectively use high-quality insights is fundamental to the discussions around usefulness of medical, digital, and pharma [1-4, 9, 13, 18]. Traditional sources such as advisory boards, MSL interactions with HCPs, publications, and congresses are themselves becoming more technologically varied, and are supplemented by new sources created by novel technologies. Making use of these myriad opportunities will require medical staff trained to recognize actionable insights, and systems that can recognize patterns. In June 2021, a new medical insight system went live. Users can input insights as free text or in response to approved surveys, record the source, associate them with predefined tags or with medical objectives, and submit them for review. Reviewers check for plausibility, quality, potential safety events, and compliance of information. A dashboard offers instant analytics and custom reports. Assessing input and addressing feedback from users are integral to the active ongoing development of the system.

\subsection{Data, Insights, and Strategy}

A major new capability established during the medical transformation is the Data, Insights \& Strategy function, incorporating the existing Biometry group alongside Epidemiology (with a new head to be recruited) and Data Strategy and RWE. This function will oversee access to advanced data sources, analytics, and machine learning and ensure a strategic approach to data and insights generation. As a first step, integrated evidence plans are being created for priority products by the end of 2021, bringing together data-generation planning across medical, health-economic outcomes research, market access, development, safety, regulatory, and commercial at a global and local level. Externally, RWE is already being used to support label expansions and approvals [19], while pharmaceutical companies and regulators are rapidly adapting to the place of RWE in drug development pathways [20, 21]. Guidelines for reporting randomized controlled trials using routinely collected data including from registries, electronic health records, and administrative databases were published in $2021 .^{22}$ While these technologybased solutions are alluring, a full appreciation of the nature of the data sources, their accuracy, completeness, and relevance are needed to allow insights and RWE to be reliably and transparently used to help bring therapeutic solutions to patients.

\subsection{Country Medical}

Worldwide, country medical health checks have been completed, resulting in profound changes in resource allocation. Investment has shifted substantially from marketing to medical, with a refocused operating model and correctly sized country medical organizations. High-value studies and programs-those most likely to make an impact for patients-have been prioritized, with support provided to rationally end underperforming studies. This step change in ambition, ensuring that all data generation activities are aligned with cross-functional plans and patient needs, aims to provide genuine and differentiated data to support better care. Involving a change in the culture of ongoing support, oversight, and performance management in relation to all forms of data generation, it has been necessary at times to shift from an activity-driven to an outcome-driven mindset, with a focus on tracking and execution across all aspects of medical and clinical operations. Ongoing efforts aim to establish a deep quality mindset and structure, develop a detailed understanding of patient flow and journey, positively influence healthcare systems to improve the standard of care, and improve knowledge transfer from headquarters to countries. Countries also offer the opportunity to pilot new approaches. For example, an innovative virtual launch event platform, hosting regional advisory boards, postlaunch events, and a homepage for medical professionals was deployed in Germany.

\section{Long-Term Follow-Up and Exploratory Analyses}

As we reach 12 months since the global launch of the medical transformation, progress has been much faster than might have been envisaged at the outset, in part owing to easier online access to stakeholders and global changes in working practices. Given the legacy of established systems and the traditional focus on developing medicines, a transformation was truly required and has been achieved, undoubtably owing to great executive support, cross-functional alignment, and a willingness to invest, as well as real engagement with workstreams at a global and local level to translate new ideas and tools into practice. A key learning was that a 
diverse range of talented cross-functional colleagues, having been invited to co-create the process and outcomes, have become enthusiastic ambassadors to their respective countries and functions, helping to embed the new culture (and preventing "not invented here" syndrome).

Nevertheless, this new structure and operating model with medical positioned and equipped to anticipate and respond to modern-day and future demands is just a beginning. Fully implementing new integrated data plans including RWE, streamlined global local translation of materials and practices, and ensuring that all new tools are implemented is a longer process. Following through on execution and metrics and ensuring that cross-functional efforts translate into improved outcomes for patients require an ongoing culture of focus, collaboration, agility, and transparency. And of course, innovation. The value of breaking silos is that everyone can learn from each other. Colleagues in research and development have been investigating synthetic trials, and medical is now exploring this area too. Machine learning has provided novel ways of understanding patient journeys and predicting patient outcomes. At the time of writing, leveraging true artificial intelligence in medical affairs remains a future prospect, but the pace of change suggests the future will be here soon, and we should be ready.

We began the transformation to place medical leadership for patients at the center of everything we do. As a dynamic process that evolves according to collective intelligence, this "transformation" must continue, but now as "how we operate". We will need to balance articulating and measuring specific goals with enabling nimble adaptation. Qualitative outcomes after another 12 months may include unanticipated innovations. The final beneficiaries of our efforts will be patients, with diseases across the TAs our specialty medical affairs teams represent. To measurably improve health outcomes for these people will require proactive internal and external sharing to implement effective strategies at the company and industry level.

\section{Conclusions and Outlook}

The strength of medical affairs has always been collaboration, trust, integrity, and transparency, providing evidencebacked information tailored to the audience. Data cannot speak for themselves. The proliferation of potential inputs and outputs has raised expectations, created a whole set of new languages for medical to translate, and necessitated a sea change not only in how resources must be marshalled but also in how the value of medical activities is demonstrated through comprehensive outcome-based metrics. Continuous leadership development is essential to ensure medical's full engagement with all stakeholders and an appropriate flow of information, and effective knowledge transfer is essential to get data to the frontline. Hopefully, our case study points to how other specialty pharma companies can leverage the advantages of a high-functioning medical organization to help drive organizational success and patient-centered outcomes.

Acknowledgements The authors thank all staff involved in the medical transformation, especially the workstream leads and partners and Executive Leadership Team members, and acknowledge support from McKinsey \& Company in the initial diagnosis phase.

\section{Declarations}

Funding Ipsen paid the open-access fee for this article. No additional funding was required.

Conflicts of interest All authors are (EL, MDA, DL, and SH) or were (AFS and AA) permanent employees of Ipsen at the time of writing. All opinions expressed in the article are those of the authors and do not necessarily represent the opinions of Ipsen.

Ethics approval Not applicable.

Consent to participate Not applicable.

Consent for publication Not applicable.

Availability of data and material Not applicable.

Code availability Not applicable

Author contributions AFS, EL, AA, MDA, DL, and SH (all authors) contributed to the concept. AFS and EL wrote the first draft. All authors discussed and contributed to the amended draft, and all approved the final version for submission. AFS, EL, and SH revised the manuscript after peer review, and all authors agree to be accountable for the work.

Open Access This article is licensed under a Creative Commons Attribution-NonCommercial 4.0 International License, which permits any non-commercial use, sharing, adaptation, distribution and reproduction in any medium or format, as long as you give appropriate credit to the original author(s) and the source, provide a link to the Creative Commons licence, and indicate if changes were made. The images or other third party material in this article are included in the article's Creative Commons licence, unless indicated otherwise in a credit line to the material. If material is not included in the article's Creative Commons licence and your intended use is not permitted by statutory regulation or exceeds the permitted use, you will need to obtain permission directly from the copyright holder. To view a copy of this licence, visit http://creativecommons.org/licenses/by-nc/4.0/.

\section{References}

1. McKinsey \& Company. A vision for medical affairs in 2025. 12 June, 2019. https://www.mckinsey.com/ /media/mckinsey/indus tries/pharmaceuticals\%20and\%20medical\%20products/our\%20ins ights/a\%20vision\%20for\%20medical\%20affairs\%20in\%202025/ a-vision-for-medical-affairs-in-2025.pdf. Accessed 11 Jan 2022. 
2. Vintura. Medical affairs in transition: towards a fully integrated model. 2019. https://www.vintura.com/wp-content/uploads/ 2019/10/Vintura-whitepaper-Medical-Affairs-in-transition.pdf. Accessed 11 Jan 2022.

3. Kinapse. Medical Affairs 2025, the future of medical affairs. 2018. https://kinapse.com/wp-content/uploads/2018/08/Kinapse-whitepaper-Medical-Affairs-2025.pdf. Accessed 11 Jan 2022.

4. McKinsey \& Company. Digital R\&D: the next frontier for biopharmaceuticals. 2017. https://www.mckinsey.com/ /media/mckin sey/industries/pharmaceuticals $\% 20$ and $\% 20$ medical $\% 20$ products/ our\%20insights/digital\%20rd\%20the $\% 20$ next $\% 20$ frontier $\% 20$ for $\%$ 20biopharmaceuticals/digitalrdthenextfrontierforbiopharma.pdf. Accessed 11 Jan 2022.

5. Logue D. A roadmap for medical affairs transformation. Journal of mHealth. 2020. https://thejournalofmhealth.com/a-roadmapfor-medical-affairs-transformation/. Accessed 11 Jan 2022.

6. Beelke ME. The evolving role of medical affairs: opportunities for discovery, preclinical and clinical research. J Clin Stud. 2017. https://www.worldwide.com/wp-content/uploads/2017/07/TheEvolving-Role-of-Medical-Affairs-Opportunities-for-DiscoveryPreclinical-and-Clinical-Research.pdf. Accessed 11 Jan 2022.

7. Muratore B. Medical affairs: the navigator of pharma's new world order. PharmaTimes magazine. 2018. http://www.pharmatimes. com/magazine/2018/julyaugust_2018/medical_affairs_the_navig ator_of_pharmas_new_world_order. Accessed 11 Jan 2022.

8. Kremer C. Why the strategic rise of medical affairs is central to pharma's future success. Medical Affairs Professional Society. 2020. https://medicalaffairs.org/charlotte-kremer-strategic-risemedical-affairs-pharma-success/. Accessed 11 Jan 2022.

9. Ghosh R, Mohanasundaram S, Shetty S, Menon S. Preparing for the next normal: transformation in the role of medical affairs following the COVID-19 pandemic. Pharmaceut Med. 2021. https:// doi.org/10.1007/s40290-021-00392-x.

10. Rajadhyaksha V. Medical affairs post-COVID 19: are we ready to take the baton? Perspect Clin Res. 2020;11(3):124-7. https://doi. org/10.4103/picr.PICR_164_20.

11. Setia S, Ryan NJ, Nair PS, Ching E, Subramaniam K. Evolving role of pharmaceutical physicians in medical evidence and education. Adv Med Educ Pract. 2018;9:777-90. https://doi.org/10. 2147/AMEP.S175683.

12. Bedenkov A, Rajadhyaksha V, Beekman M, Moreno C, Fong P-C, Agustin L, Odell S. Developing medical affairs leaders who create the future. Pharmaceut Med. 2020;34(5):301-7. https://doi.org/10. 1007/s40290-020-00351-y.

13. Bedenkov A, Moreno C, Agustin L, Jain N, Newman A, Feng L, Kostello G. Customer centricity in medical affairs needs human-centric artificial intelligence. Pharmaceut Med. 2021;35(1):21-9. https://doi.org/10.1007/s40290-020-00378-1.

14. Kremer CME, Piliero PJ, du Plessis D. Communicating the value of medical affairs. A MAPS White Paper. Medical Affairs Professional Society. 2020. https://medicalaffairs.org/communicatingthe-value-of-medical-affairs/. Accessed 11 Jan 2022.

15. Harmon B, Tebbey P, Walz A, Williams L. The value and impact of medical affairs: mastering the art of leveraging meaningful metrics. A MAPS White Paper. Medical Affairs Professional Society. 2021. https://medicalaffairs.org/wp-content/uploads/2021/07/ White-Paper-The-Art-Of-Metrics-FINAL.pdf. Accessed 11 Jan 2022.

16. Horwood C, Stiewe S. Medical affairs: the future strategists of the pharma industry? 2021. https://uptakestrategies.com/medical-affai rs-the-future-strategists-of-the-pharma-industry/. Accessed 11 Jan 2022.

17. SVP Medical, Sanofi. The future of medical affairs: a conversation with Beat Sümegi. Medical Affairs Professional Society. 2020. https://medicalaffairs.org/future-medical-affairs-beat-sumegisanofi/. Accessed 11 Jan 2022.

18. McLoughlin M, Jonkman M, Zivkov M. Moving to an integrated, holistic approach to insights-related activities in medical affairs. A MAPS White Paper. Medical Affairs Professional Society. 2021. https://medicalaffairs.org/moving-to-an-integrated-holistic-appro ach-to-insights-related-activities-in-medical-affairs/. Accessed 11 Jan 2022.

19. Wakefield P, François C, Leray A, Hartry A. Essentials of realworld evidence. Medical Affairs Professional Society. 2021. https://medicalaffairs.org/essentials-real-world-evidence/. Accessed 11 Jan 2022.

20. Marquis-Gravel G, Roe MT, Turakhia MP, et al. Technology-enabled clinical trials: transforming medical evidence generation. Circulation. 2019;140(17):1426-36. https://doi.org/10.1161/CIRCU LATIONAHA.119.040798.

21. Khosla S, White R, Medina J, Ouwens M, Emmas C, Koder T, et al. Real world evidence (RWE): a disruptive innovation or the quiet evolution of medical evidence generation? F1000Res. 2018;7:111. https://doi.org/10.12688/f1000research.13585.2.

22. Kwakkenbos L, Imran M, McCall SJ, et al. Consort extension for the reporting of randomised controlled trials conducted using cohorts and routinely collected data (CONSORT-ROUTINE): checklist with explanation and elaboration. BMJ. 2021;373:n857. https://doi.org/10.1136/bmjopen-2021-049093. 